\title{
Autoantibody-mediated desialylation impairs human thrombopoiesis and platelet lifespan
}

Haematologica 2021

Volume 106(1):196-207

\section{Correspondence:}

TAMAM BAKCHOUL

tamam.bakchoul@med.uni-tuebingen.de

Received: August 26, 2019.

Accepted: December 11, 2019.

Pre-published: December 19, 2019.

https://doi.org/10.3324/haematol.2019.236117

(C)2021 Ferrata Storti Foundation

Material published in Haematologica is covered by copyright. All rights are reserved to the Ferrata Storti Foundation. Use of published material is allowed under the following terms and conditions:

https://creativecommons.org/licenses/by-nc/4.0/legalcode. Copies of published material are allowed for personal or internal use. Sharing published material for non-commercial purposes is subject to the following conditions:

https://creativecommons.org/licenses/by-nc/4.0/legalcode, sect. 3. Reproducing and sharing published material for commercial purposes is not allowed without permission in writing from the publisher.
Irene Marini, ${ }^{1}$ Jan Zlamal, ${ }^{1}$ Christoph Faul, ${ }^{2}$ Ursula Holzer, ${ }^{3}$ Stefanie Hammer, ${ }^{4}$ Lisann Pelzl, ${ }^{1}$ Wolfgang Bethge, ${ }^{2}$ Karina Althaus ${ }^{1,4}$ and Tamam Bakchoul ${ }^{1,4}$

${ }^{1}$ Transfusion Medicine, Medical Faculty of Tübingen, University Hospital of Tübingen; ${ }^{2}$ Department of Internal Medicine II, Oncology, Hematology, Clinical Immunology, Rheumatology and Pneumology, University Hospital of Tübingen; ${ }^{3}$ Department of Pediatric Hematology and Oncology, University Children's Hospital of Tübingen and ${ }^{4}$ Center for Clinical Transfusion Medicine, University Hospital of Tübingen, Tübingen, Germany

\section{ABSTRACT}

Tmmune thrombocytopenia is a common bleeding disease caused by autoantibody-mediated accelerated platelet clearance and impaired thrombopoiesis. Accumulating evidence suggests that desialylation affects platelet lifespan in immune thrombocytopenia. Herein, we report on novel effector functions of autoantibodies from patients with immune thrombocytopenia, which might interfere with the clinical picture of the disease. Data from our study show that a subgroup of autoantibodies is able to induce cleavage of sialic acid residues from the surface of human platelets and megakaryocytes. Moreover, autoantibody-mediated desialylation interferes with the interaction between cells and extracellular matrix proteins leading to impaired platelet adhesion and megakaryocyte differentiation. Using a combination of an ex vivo model of thrombopoiesis, a humanized animal model, and a clinical cohort study, we demonstrate that cleavage of sialic acid induces significant impairment of the production, survival as well as function of human platelets. These data may indicate that prevention of desialylation should be investigated in the future in clinical studies as a potential therapeutic approach to treat bleeding in immune thrombocytopenia.

\section{Introduction}

Immune thrombocytopenia (ITP) is an autoimmune disorder characterized by bleeding due to isolated thrombocytopenia with a platelet count below $100 \times 10^{9} / \mathrm{L} .1,2$ The incidence of ITP ranges between 3.3-3.9/100,000 per year in adults, and between 1.9-6.4/100,000 per year in children. ${ }^{3,4}$ While the disorder has a brief course with spontaneous remission in the majority of children, most adult patients develop chronic ITP which can be associated with clinically significant bleeding including hemorrhages in the skin or mucous membranes, giving rise to petechiae and purpura and, rarely, intracranial manifestations. ${ }^{5,6}$ The exact mechanism of autoimmunity leading to ITP is still unclear, but includes an alteration of the balance between effector and regulatory cells. ${ }^{5}$ This imbalance results in a breakdown of the immune tolerance in ITP patients, causing functional alterations in the humoral and cellular immune systems which are thought to contribute to the pathogenesis of ITP. For a long time, it was thought that the low platelet count is caused solely by enhanced destruction of opsonized platelets in the spleen upon binding of the antiplatelet autoantibodies $(\mathrm{AAb})$ to the glycoproteins expressed on the surface of platelets. ${ }^{6.8}$ However, platelet desialylation has recently been suggested to be an alternative mechanism of platelet destruction, which occurs in the liver through AshwellMorell receptors. ${ }^{9}$ This may explain the observation that a subgroup of ITP patients (up to $20 \%$ ) is refractory to splenectomy and treatment with intravenous immune globulin $\mathrm{G}(\operatorname{IgG}) .^{10}$ Furthermore, in recent years more attention has been drawn to impaired platelet generation as another major cause of the low platelet count in ITP.,12 This is due to the fact that megakaryocytes, the cells that release platelets into the circulation, ${ }^{13}$ express the same glycoproteins (GPIIb/IIa and GPIb/IX), present on the platelet's surface, that are targeted by AAb in ITP..$^{12,14}$ 
Based on these considerations, we hypothesized that the AAb-mediated desialylation of the glycoproteins expressed on platelets and megakaryocytes may interfere with platelet lifespan, affecting the clinical picture in ITP. In this study, we investigated a cohort of ITP patients with a focus on the impact of AAb-mediated desialylation on the survival and function of human platelets and megakaryocytes.

\section{Methods}

\section{Study cohort}

Patients were enrolled into the study after a retrospective review of their clinical records by two physicians to confirm the diagnosis of ITP. Experiments were performed using leftover serum from the ITP patients. Control sera were obtained from non-ITP thrombocytopenic patients (with hypoproliferative thrombocytopenia) as well as from randomly chosen healthy blood donors, after obtaining written consent. Studies involving human material were approved by the Ethics Committee of the Medical Faculty, Eberhard-Karls Universität, University Hospital of Tübingen, Germany, and were conducted in accordance with the Declaration of Helsinki. Additional details are available in the Online Supplementary Methods.

\section{Antibody characterization}

Free and platelet-bound AAb were detected by the monoclonal antibody-specific immobilization of platelet antigens (MAIPA) assay, as described previously. ${ }^{15-17}$ AAb-mediated changes in the sialylation status of platelets and megakaryocytes were analyzed using a flow cytometer. In order to investigate the AAb-induced apoptosis, the mitochondrial inner transmembrane potential was measured by flow cytometry, as previously described with minor modifications..$^{18}$ Furthermore, the impact of desialylation on the ability of platelets to adhere to human serum albumin (Kedrion, Barga, Italy), fibrinogen (Sigma Aldrich, Munich, Germany) or von Willebrand factor (VWF: Baxalta, Vienna, Austria) was assessed using an adhesion test, as previously described. ${ }^{19}$ Additional information is provided in the Online Supplementary Methods and Online Supplementary Figure S1.

\section{In vivo analysis}

The impact of desialylation on the survival of human platelets was analyzed using the NSG mouse model, as described previously. ${ }^{20,21}$ All animal studies were conducted in Tübingen, Germany, and were approved by the responsible authorities of the State of Baden-Württemberg.

\section{In vitro generation of human megakaryocytes}

Megakaryocytes were produced from $\mathrm{CD} 34^{+}$cells as previously described with minor modifications. ${ }^{22,23}$ In brief, CD $34^{+}$cells were magnetically isolated and cultured for differentiation into megakaryocytes in cell medium supplemented with $50 \mathrm{ng} / \mathrm{mL}$ of thrombopoietin for 14 days. The megakaryocytes generated in vitro were identified and characterized using a flow cytometry gating strategy based on DNA content, expression of CD41 and CD42a as well as viable markers, as previously described. ${ }^{23,24}$ In order to investigate the impact of AAb-induced desialylation on thrombopoiesis, megakaryocytes were incubated with IgG from ITP patients or healthy donors and proplatelet generation as well as platelet release were assessed by microscopy and flow cytometry, respectively, as previously described with minor modifications. ${ }^{25}$ Furthermore, the ability of megakaryocytes to adhere to human serum albumin, fibrinogen, VWF or collagen (Collagen-
Horm, Takeda, Linz, Austria) was verified and quantified after 2 hours (h) of incubation with IgG fractions from ITP patients or healthy donors, taking images from seven different microscope fields (x40, Olympus IX73, Olympus GmbH, Germany). Additional details are provided in the Online Supplementary Methods and Online Supplementary Figure S2.

\section{Statistical analysis}

Statistical analyses were performed using GraphPad Prism 7 (La Jolla, CA, USA). A $t$-test was used to analyze normally distributed data, whereas a nonparametric test (Mann-Whitney test) was used when data were not normally distributed, as assessed by the D'Agostino and Pearson omnibus normality test. Group comparisons were performed using the Wilcoxon rank-sum test and the Fisher exact test with categorical variables. A $P$-value $<0.05$ was assumed to represent statistical significance.

\section{Results}

\section{Patients' characteristics}

The clinical records of 100 patients with ITP were independently reviewed by two physicians. Forty percent of the patients were female and the median age at the time that laboratory testing was performed was 54 years (interquartile range [IOR], 0-86 years) (Table 1). The median platelet count was $32 \times 10^{9} / \mathrm{L}$ (IOR, $7-74 \times 10^{\circ} / \mathrm{L}$ ) and $48 / 100$ (48\%) patients reported bleeding events. With regards to therapy, $35 / 100(35 \%)$ patients had received steroids and $32 / 100$ (32\%) had been treated with high doses of intravenous IgG. Rituximab and thrombopoietin analogs were given as second-line treatments to seven $(7 \%)$ and eight ( $8 \%)$ patients, respectively. On the day of laboratory investigation, MAIPA assay revealed positive results for free or bound $\mathrm{AAb}$ in 51/100 (51\%) patients, while no AAb were detectable in the sera of 49/100 (49\%) patients (Table 1 and Online Supplementary Table S1).

\section{Prevalence and clinical relevance of desialylating autoantibodies}

According to the calculated cutoffs (fold increases of 1.75 and 1.42 , for $\beta$-galactose and $\mathrm{N}$-acetylglucosamine, respectively) sera from 35/100 ITP patients of the study cohort induced desialylation. In particular, sera from 28/100 (28\%) patients caused the exposure of $\beta$-galactose, sera from $21 / 100(21 \%)$ caused the exposure of $\mathrm{N}$-acetylglucosamine, and sera from 14/100 (14\%) led to the exposure of both residues (Figure 1A, B). A comparable frequency of desialylating $\mathrm{AAb}$ was observed between pediatric and adult patients (39\% vs. $34 \%$, respectively; $P=0.8037$ ). Furthermore, more sera from patients with secondary ITP induced desialylation in comparison to sera from patients with primary ITP ( $60 \%$ vs. $31 \%$, respectively; $P=0.0395)$.

Lower platelet counts were documented in patients with $\mathrm{AAb}$ inducing desialylation compared to those with no desialylating ability (median [IOR], $13 \times 10^{9} / \mathrm{L}$ [5$\left.42 \times 10^{9} / \mathrm{L}\right]$ vs. $46 \times 10^{9} / \mathrm{L} \quad\left[11-81 \times 10^{9} / \mathrm{L}\right]$, respectively; $P=0.0286$ ) (Table 1). Most importantly, bleeding events were more frequently reported by patients with desialylating $\mathrm{AAb}(72 \%$ vs. $28 \% ; P=0.0008)$ (Table 1). In particular, a greater tendency to develop petechiae (46\% vs. $19 \% ; P=0.0044)$ and hematomas (49\% vs. $17 \%$; $P=0.0009)$ was observed in ITP patients with desialylating $\mathrm{AAb}$ compared to that in patients who tested negative in the lectin-binding assay. 
A

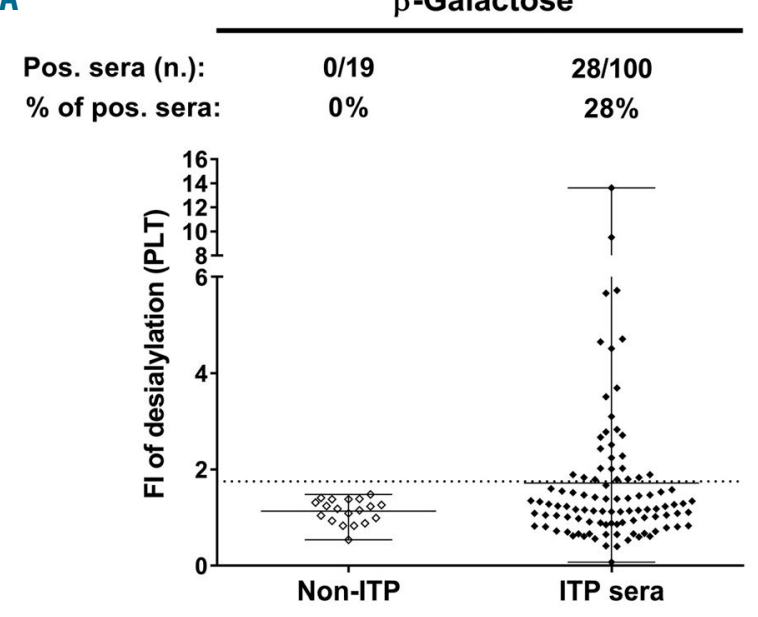

C

$\begin{array}{lllll}\text { Pos. sera (n.): } & 3 / 5 & 11 / 23 & 7 / 23 & 7 / 49 \\ \text { \% of pos. sera: } & 60 \% & 48 \% & 30 \% & 14 \%\end{array}$

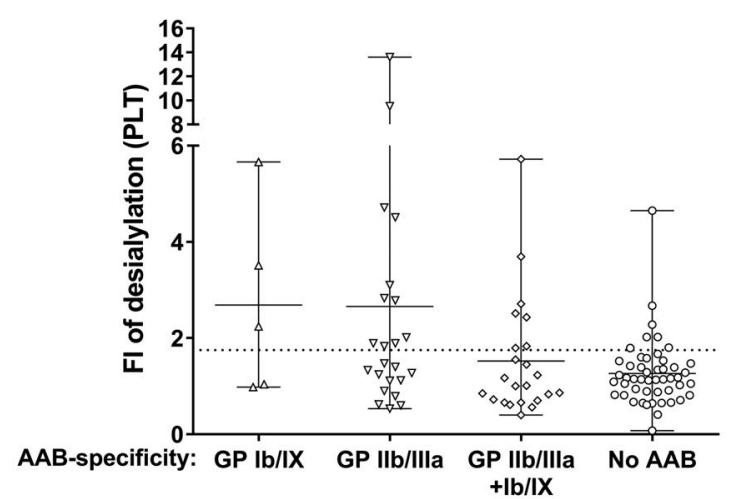

B

N-Acetylglucosamine

$\begin{array}{lcc} & 0 / 19 & 21 / 100 \\ \text { Pos. sera (n.): } & 0 \% & 21 \% \\ \text { \% of pos. sera: } & & \end{array}$

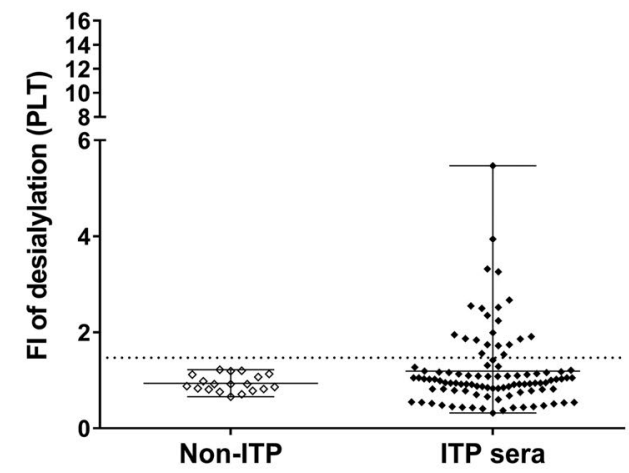

D

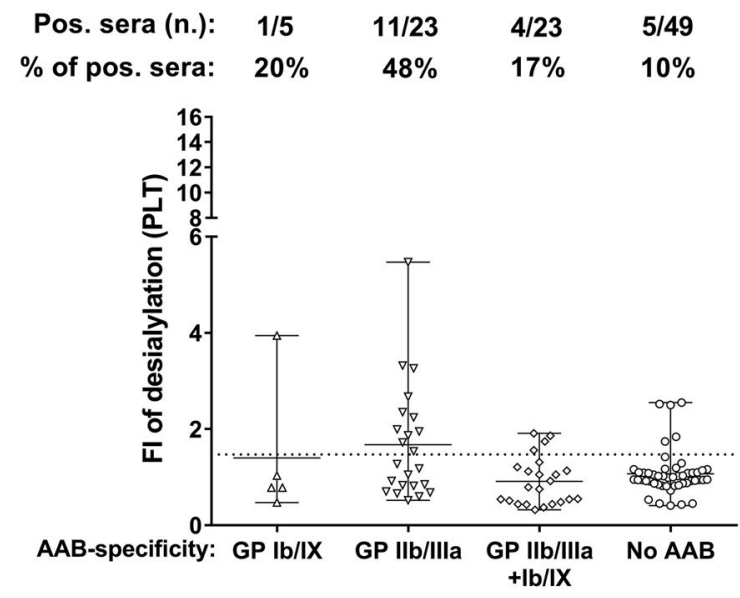

Figure 1. Platelet desialylation ability of autoantibodies from patients with immune thrombocytopenia and the impact of glycoprotein specificity. (A, B) Sera from thrombocytopenic patients without immune thrombocytopenia (ITP, empty diamonds) and patients with ITP (black diamonds) were incubated with washed platelets from healthy donors. Cleavage of sialic acid and exposure of $\beta$-galactose (A) and $N$-acetylglucosamine (B) on test platelets was detected by flow cytometry and determined as fold increase of the cleavage compared to that when the platelets were incubated with control sera. (C, D) Distribution of desialylating autoantibodies according to the glycoprotein specificity as determined in a monoclonal antibody-specific immobilization of platelet antigens (MAIPA) assay. Data are presented as the mean of the fold increase for each sample when tested with platelet suspensions from three healthy donors and compared to control sera. The number of ITP sera tested is reported in the graphs. The values presented are the mean and range. FI: fold increase; PLT: platelet; AAB: autoantibody; GP: glycoprotein.

The impact of glycoprotein specificity on the ability to cleave sialic acid

Among the 51 patients with a positive test result in the MAIPA assay for at least one glycoprotein, desialylation was observed in 26 (51\%). Not only did sera and IgG containing $\mathrm{AAb}$ against GPIb/IX cause platelet desialylation, but those containing anti-GPIIb/IIIa AAb were also able to induce cleavage of sialic acid and expose $\beta$-galactose or $\mathrm{N}$-acetylglucosamine $(60 \%$ vs. $48 \%$ and $20 \%$ vs. $48 \%$, respectively) (Figure $1 \mathrm{C}, \mathrm{D})$. Of note, cleavage of sialic acid was also observed for sera without detectable AAb in the MAIPA assay, leading to increased expression of $\beta$ galactose and $\mathrm{N}$-acetylglucosamine in $7 / 49(14 \%)$ and $5 / 49(10 \%)$ cases, respectively (Figure 1C, D). This may indicate the presence of low-avidity $\mathrm{AAb}$ in these sera, as has been reported previously for anti-HPA-1a alloantibodies $^{26,27}$ and complement-fixing AAb. ${ }^{28}$

\section{Autoantibodies against GPIIb/IIla induce desialylation} via crosslinking Fc $\gamma$ RIIA

It is well established that AAb against GPIb/IX cause platelet desialylation via Fc-independent release of sialidase from $\alpha$ granules of platelets. ${ }^{9}$ To verify the mechanism of platelet desialylation by sera containing antiGPIIb/IIIa AAb, the expression of $\beta$-galactose and $\mathrm{N}$ acytelglucosamine was analyzed after incubation of washed platelet with IgG fractions from these sera. No significant difference in the ability to induce cleavage of sialic acid from the platelet surface was observed between patients' sera and their corresponding IgG fractions (fold-increase, [mean \pm standard error of mean, SEM]: $5.96 \pm 1.31$ vs. $5.69 \pm 0.99 ; P=0.8714$ and $3.69 \pm 0.76$ vs. $5.20 \pm 0.21, P=0.1028$, for $\beta$-galactose and $\mathrm{N}$-acetylglucosamine, respectively) (Figure $2 \mathrm{~A}, \mathrm{~B}$ ). These results indicate that IgG AAb initiate the cleavage of the sialic acid. Next, washed platelets were preincubated with sialidase inhibitor. A significant reduction of the IgG-mediated cleavage of sialic acid was observed ( $\%$ desialylation $[$ mean \pm SEM]: $100 \pm 0 \%$ vs. $63 \pm 2 \% ; P=0.0001$ and $100 \pm 0 \%$ vs. $57 \pm 6 \% ; P=0.0002$, for $\beta$-galactose and $N$ acetylglucosamine, respectively) (Figure $2 \mathrm{C}, \mathrm{D})$. Since the experiments were performed with IgG fractions and 
Table 1. Demographic characteristics and clinical manifestations of the study cohort.

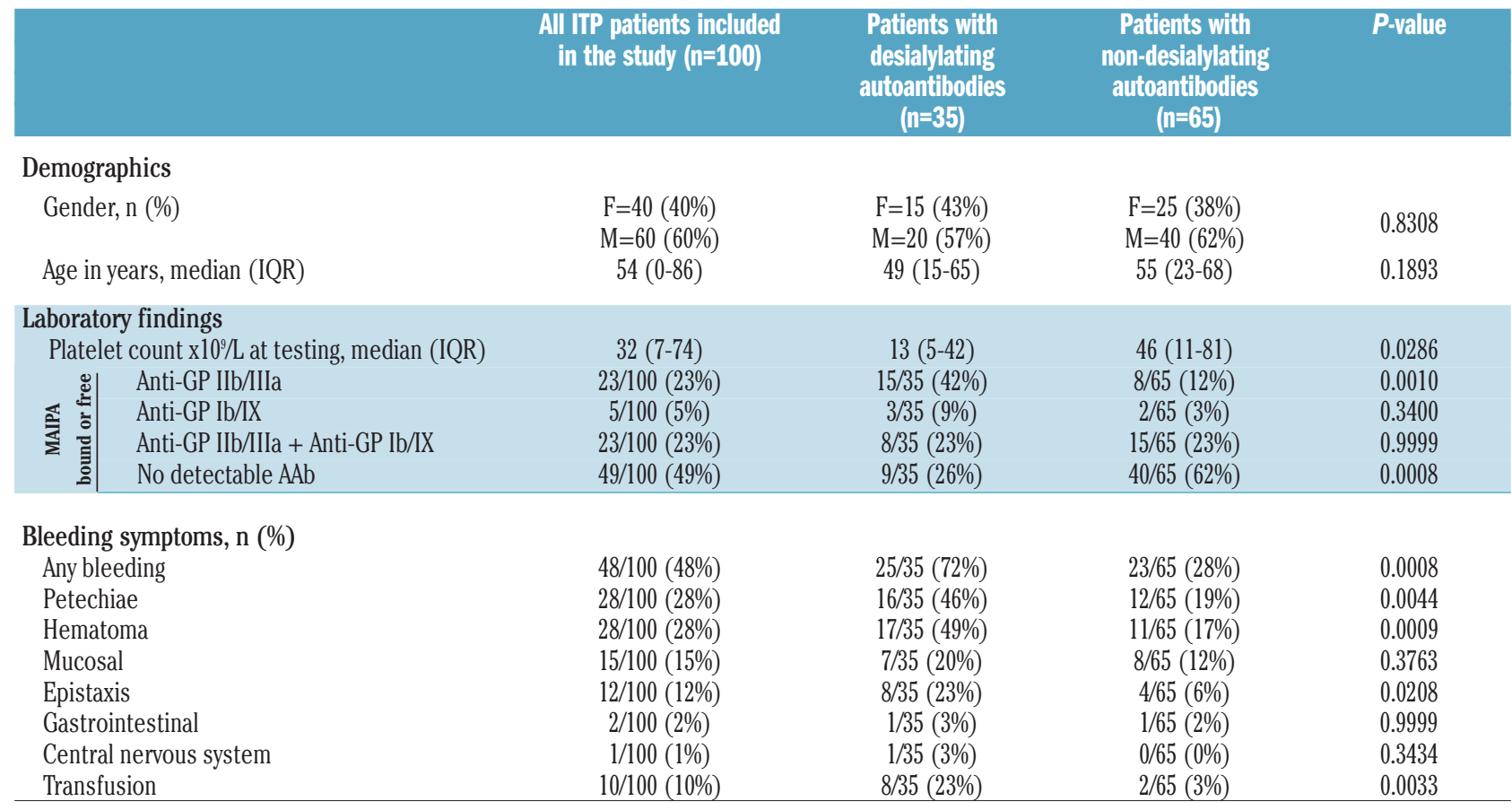

* Results of antibody testing are reported as positive when either the direct or indirect monoclonal antibody-specific immobilization of platelet antigens (MAIPA) assay revealed a positive reaction, indicating the presence of platelet-bound and free autoantibodies, respectively. F: female; M: male; IQR: interquartile range; GP: glycoprotein; AAb: autoantibodies.

washed platelets, these data suggest that intracellular and not plasma sialidase is responsible for the AAb-mediated desialylation of platelets. Next, we blocked the Fcy RIIA signaling using the monoclonal antibody IV.3. Significant decreases in the expression of $\beta$-galactose and $\mathrm{N}$-acetylglucosamine were observed (\% desialylation [mean \pm SEM]: $100 \pm 0 \%$ vs. $52 \pm 11 \% ; P=0.0110$ and $100 \pm 0 \%$ vs. $60 \pm 9 \% ; P=0.0190$, for $\beta$-galactose and $N$-acetylglucosamine, respectively) (Figure 2E, F). Taken together, these results indicate that IgG AAb against GPIIb/IIIa are able to crosslink Fc $\gamma$ RIIA leading to the release of sialidase from the intracellular compartments of platelets.

\section{Desialylation contributes to antibody-mediated destruction of human platelets}

Patients with desialylating AAb had significantly lower platelet counts compared to patients with sera that failed to induce cleavage of sialic acid (median platelet count [range], $13 \times 10^{9} / \mathrm{L}\left[0-106 \times 10^{9} / \mathrm{L}\right]$ vs. $46 \times 10^{9} / \mathrm{L}\left[1-234 \times 10^{9} / \mathrm{L}\right]$; $P=0.0121$ ) (Figure 3A). A NSG mouse model was used to analyze the impact of $\mathrm{AAb}$ inducing desialylation on human platelet survival. In this model, $80-100 \times 10^{6} / \mu \mathrm{L}$ human platelets ( $8-10 \%$ of the total circulating platelets) are usually detectable 30 minutes (min) after injection into the murine circulation, and more than $70 \%$ of these platelets circulate for up to $5 \mathrm{~h}$ in the absence of plateletreactive antibodies (data not shown). Upon injection of IgG fractions containing desialylating AAb from ITP patients, a rapid reduction of platelet survival was observed compared to that following injection of control IgG $(\%$ human platelet survival after $5 \mathrm{~h}$ [mean \pm SEM]: $28 \pm 5 \%$ vs. $79 \pm 5 \%$, respectively; $P=0.0005$ ) (Figure $3 \mathrm{~B}$ ). AAbmediated platelet clearance was partially dependent on desialylation. In fact, in the presence of a sialidase inhibitor a remarkable rescue of platelet survival was observed (\% human platelet survival after $5 \mathrm{~h}$ [mean \pm
SEM]: $28 \pm 5 \%$ vs. $45 \pm 3 \%$; $P=0.0191$ ) (Figure 3B). These results indicate that the ability to induce cleavage of sialic acid contributes to AAb-mediated platelet destruction in vivo.

\section{Antibody-mediated desialylation is not associated with platelet apoptosis}

To verify a potential correlation between platelet desialylation and apoptosis, mitochondrial inner transmembrane potential depolarization was investigated after incubation with desialylating and non-desialylating AAb. No correlation was observed between the ability of $\mathrm{AAb}$ to induce desialylation and apoptosis $\left(r^{2}:-0.333, P=0.3510\right.$ vs. $\mathrm{r}^{2}-0.344, P=0.3310$, for $\beta$-galactose and $\mathrm{N}$-acetylglucosamine, respectively) (Figure $3 \mathrm{C}, \mathrm{D})$.

\section{Desialylation contributes to autoantibody-mediated platelet dysfunction}

By testing IgG fractions from ITP patients, it was seen that inhibition of platelet adhesion was greater with desialylating $\mathrm{AAb}$ than with non-desialylating ones (\% adherent cells/field [mean \pm SEM]: $74 \pm 8 \%$ vs. $34 \pm 7 \%$; $P=0.0021$ and $67 \pm 5 \%$ vs. $26 \pm 2 \% ; P=0.0027$, for fibrinogen and VWF, respectively) (Figure 4A, B). When platelets were pretreated with a pharmaceutically available sialidase inhibitor, significant improvements of platelet adhesion were observed ( $\%$ adherent cells/field [mean \pm SEM]: $36 \pm 5 \%$ vs. $86 \pm 6 \% ; P=0.0001$ and $26 \pm 2 \%$ vs. $67 \pm 11 \% ; P=0.0203$, for fibrinogen and VWF, respectively) (Figure 4C, D).

\section{Desialylating autoantibodies impair in vitro thrombopoiesis}

To determine the ability of AAb to induce desialylation of glycoproteins on the surface of megakaryocytes, IgG fractions from ten ITP sera that induced a change in the 
A

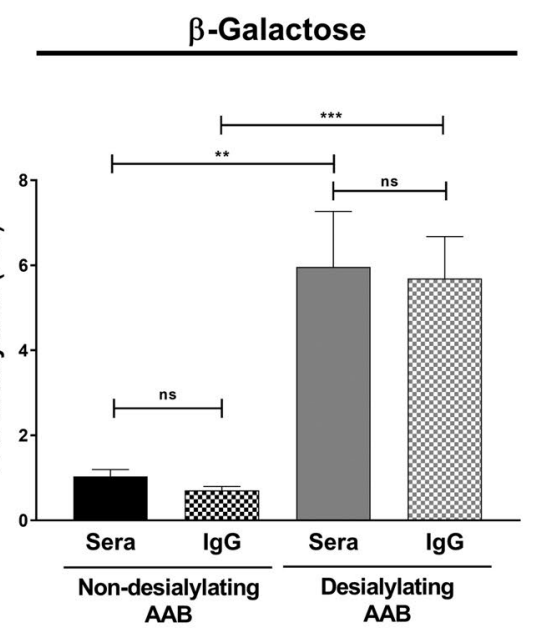

C

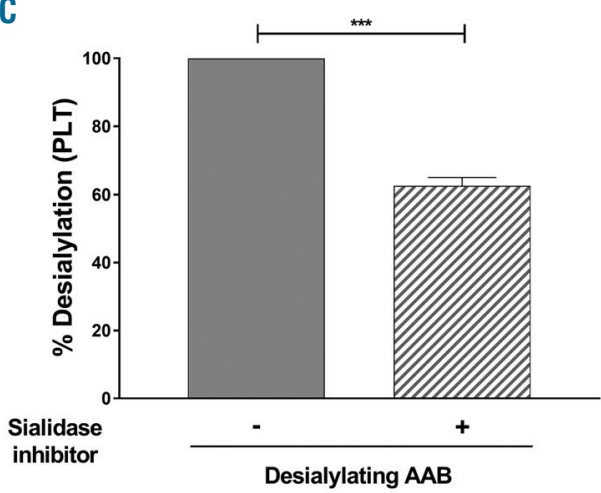

E

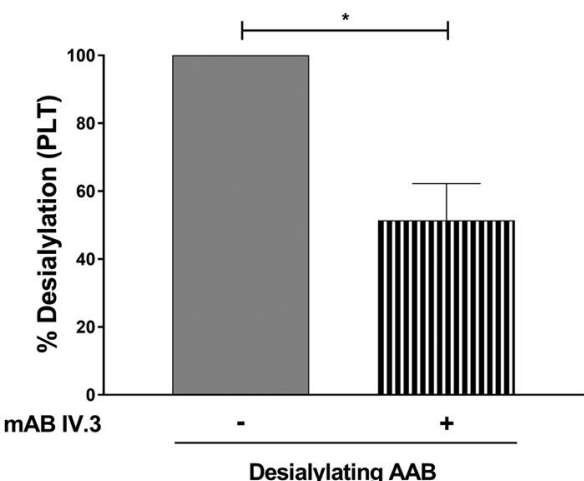

B

N-Acetylglucosamine
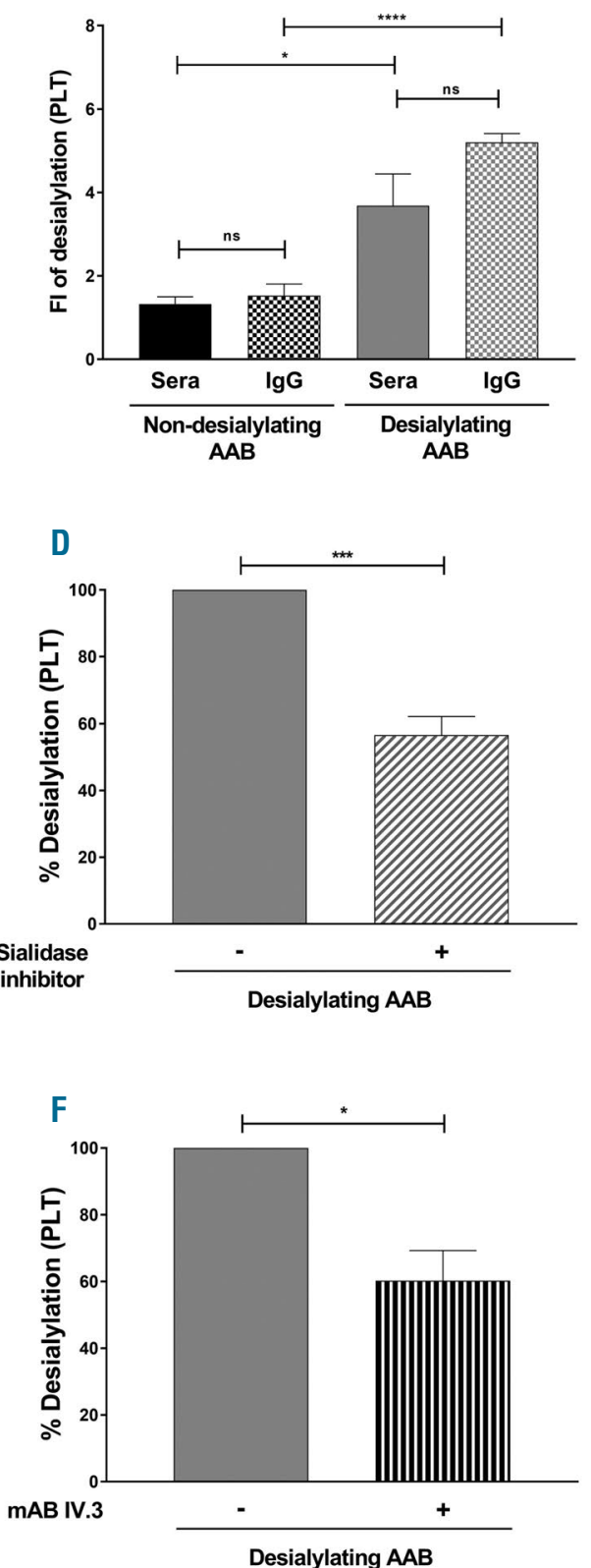

Figure 2. Mechanism of antiGPIIb/IIla-mediated platelet desialylation. Platelets from healthy donors were incubated with sera or immunoglobulin $\mathrm{G}$ (IgG) fractions from patients with immune thrombocytopenia (ITP) with non-desialylating (black columns) and desialylating (gray columns) autoantibodies (AAB). Cleavage of sialic acid and exposure of $\beta$-galactose (A) and N-acetylglucosamine (B) on test platelets was detected by flow cytometer. Note that there was no difference in the desialylation ability between sera and corresponding IgG fractions. (C-F) To verify the mechanism of platelet desialylation induced by antiGPIIb/IIla AAB, platelets were preincubated with buffer or a sialidase inhibitor (C,D), or the monoclonal antibody IV.3 (E, F) prior to the addition of IgG fractions from sera containing desialylating $A A B$ against GPIIb/IIla complexes. IgG from three different ITP patients were tested in four independent experiments using washed platelets from healthy donors. Data are presented as the mean \pm standard error of mean of the measured fold increase compared to the data obtained for IgG from three healthy donors used as controls. ns, not significant, $\quad * P<0.05$, $* * P<0.01, \quad * * * P<0.001$ and $* * * * P<0.0001$. Fl: fold increase; PLT: platelet; GP: glycoprotein; mAB: monoclonal antibody. sialylation pattern on platelets were incubated with megakaryocytes derived from peripheral blood CD34+ cells. As shown in Figure 5A, IgG fractions from eight of the ten sera were able to induce cleavage of sialic acid and lead to exposure of $\beta$-galactose and $\mathrm{N}$-acetylglucosamine. More interestingly, the ability to initiate desialylation was not restricted to $\mathrm{AAb}$ against GPIb/IX. In fact, anti-GPIIb/IIIa $\mathrm{AAb}$ were also able to induce megakaryocyte desialylation (Figure 5B, C).

Next, we investigated the impact of AAb-mediated desialylation on proplatelet formation. Megakaryocytes that were cultured in the presence of IgG fractions from desialylating $\mathrm{AAb}$ showed significantly less ability to form proplatelet extensions compared to those cultured in the presence of control IgG ( $\%$ proplatelet-forming megakaryocytes, [mean \pm SEM]: $100 \pm 0 \%$ vs. $42 \pm 12 \% ; P=0.0037$ )
(Figure 6A). More interestingly, the ability to form proplatelets was largely rescued when sialidase was inhibited (\% proplatelet-forming megakaryocytes [mean \pm SEM]: $42 \pm 12 \%$ vs. $90 \pm 9 \% ; P=0.0326$ ) (Figure 6A-C).

\section{Autoantibody-mediated desialylation interferes with the interaction between megakaryocytes and extracellular matrix proteins}

Interaction between megakaryocytes and extracellular matrix proteins was reported to be essential for the differentiation of these cells and their ability to form proplatelet extensions. ${ }^{29}$ When megakaryocytes were incubated with desialylating $\mathrm{AAb}$, it was found that there was a significant reduction of cells that adhered to fibrinogen and VWF, but not collagen, compared to when the megakaryocytes were incubated with non-desialylating 
A

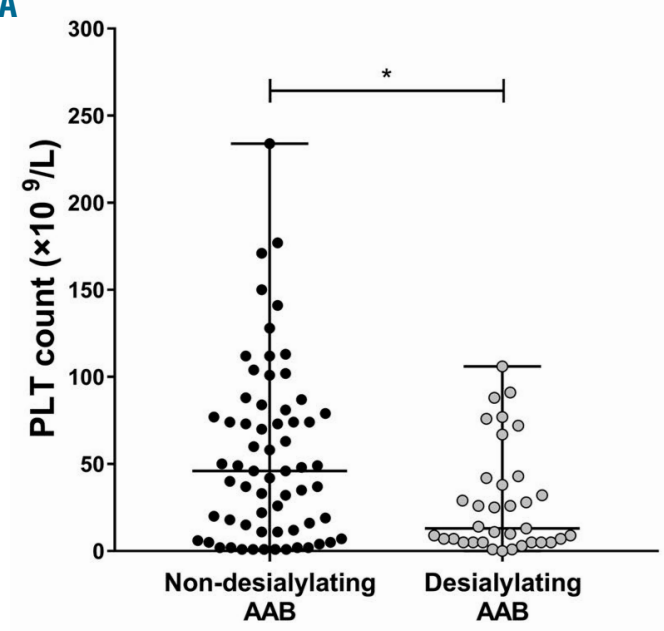

C

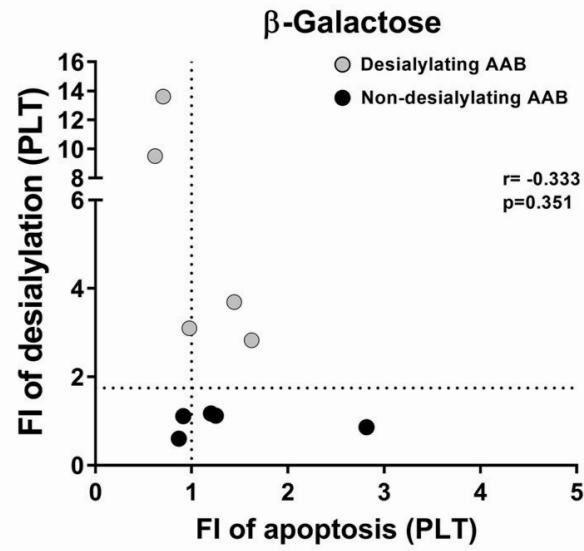

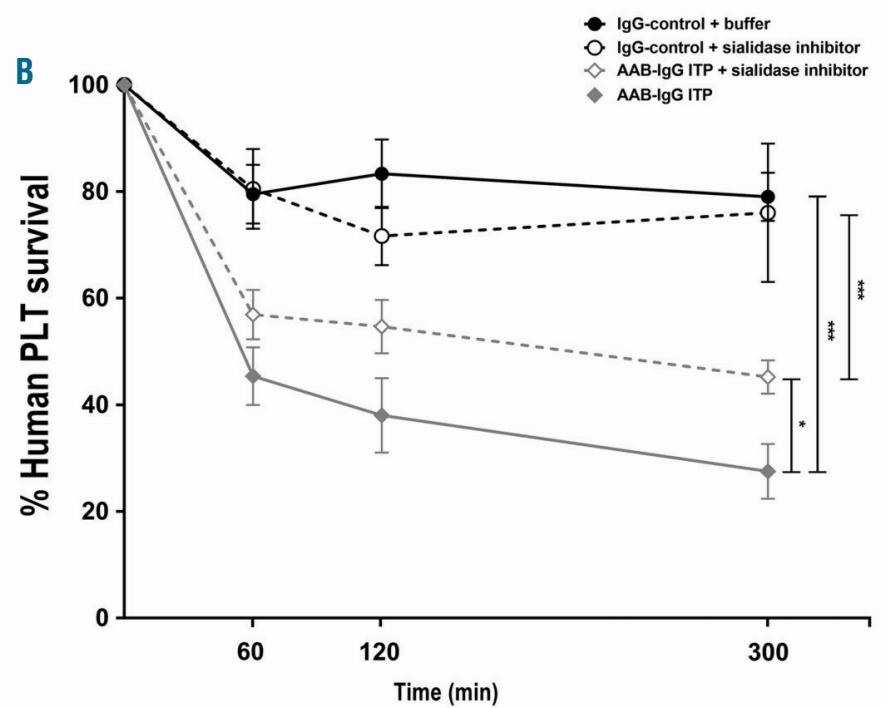

D

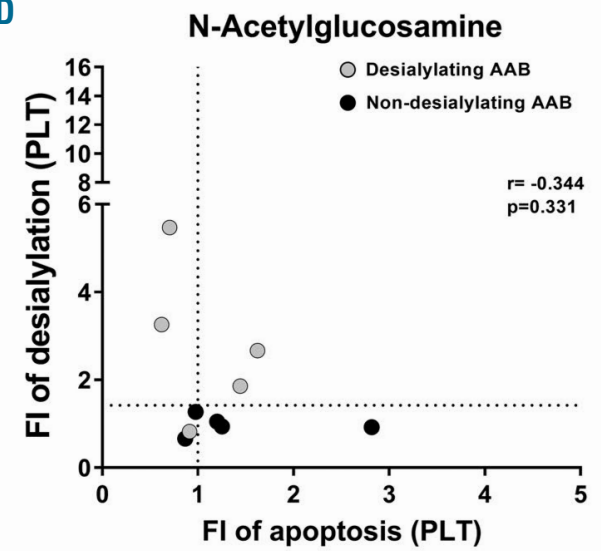

Figure 3. The impact of desialylating autoantibodies on human platelet survival. (A) Platelet count was determined on the day of sample collection for laboratory investigations for patients with immune thrombocytopenia (ITP) with non-desialylating (black circles) and desialylating (gray circles) autoantibodies (AAB). Symbols represent the platelet count of each patient. Lines indicate the median and range of platelet counts in each group, tested in four different experiments using washed platelets from three different healthy donors. (B) The direct impact of desialylating AAB on platelet survival was analyzed using the NSG mouse model. Freshly isolated human platelets were treated with buffer (filled symbols) or a sialidase inhibitor (empty symbols) and injected into NGS mice via the lateral tail vein. After 30 minutes (min), blood was taken to determine the baseline value of circulating human platelets (100\%). Subsequently, immunoglobulin G (IgG) fractions of desialylating ITP sera (gray lines) or sera from healthy donors (black lines) were injected and further blood samples were taken at 60, 120, and 300 min after baseline, as indicated. The data shown are the mean percentage \pm standard error of mean of the remaining human platelets in the mouse circulation after injection of four different AAB. (C, D) The correlation between the ability of AAB to induced desialylation and apoptosis was investigated by incubating lgG fractions from non-desialylating (black circles) and desialylating (gray circles) AAB with washed platelets from healthy donors and exposure of $\beta$-galactose (C) and N-acetylglucosamine (D), and changes in mitochondrial inner transmembrane potential were analyzed. Spearman $r^{2}$ : $-0.333, P=0.3510$ vs. $r^{2}$ :-0.344, $P=0.3310$, for $\beta$-galactose and $N$-acetylglucosamine, respectively. Data are presented as mean of the fold increase evaluated in three different experiments using washed platelets from three healthy donors. ${ }^{*}<0.05$ and $* * * P<0.001$. PLT: platelet; Fl: fold increase.

$\mathrm{AAb}(\%$ adherent cells/field [mean $\pm \mathrm{SEM}]: 88 \pm 8 \%$ vs. $58 \pm 8 \%, P=0.0479 ; 79 \pm 8 \%$ vs. $47 \pm 4 \%, P=0.0232$ and $90 \pm 5 \%$ vs. $88 \pm 4 \%, P=0.8080$, for fibrinogen, VWF and collagen, respectively) (Figure 7A-C). This effect was dependent on the ability of the AAb to induce desialylation. In fact, in the presence of a sialidase inhibitor there were significant improvements of the ability of megakaryocytes to adhere to both proteins (\% adherent cells/field [mean \pm SEM]: $50 \pm 6 \%$ vs. $88 \pm 5 \%, P=0.0022$ and $47 \pm 4 \%$ vs. $82 \pm 7 \%, P=0.0020$, for fibrinogen and VWF, respectively) (Figure 7D, E). In contrast, no difference was observed for cell adhesion to collagen (\% adherent cells/field [mean \pm SEM]: $90 \pm 4 \%$ vs. $88 \pm 6 \%$; $P=0.7083$ ) (Figure 7F).

To confirm that sialylation plays an important role in megakaryocyte differentiation, exogenous sialidase was added to the culture medium at the beginning of the differentiation stage. As shown in Online Supplementary Figure S3, the numbers of total megakaryocytes as well as mature megakaryocytes were lower in the presence of sialidase than in the presence of buffer $\left(\mathrm{CD} 41^{+}\right.$megakaryocytes $/ \mu \mathrm{L}$ $[$ mean \pm SEM] $: 69 \pm 11$ cells $/ \mu \mathrm{L}$ vs. $27 \pm 4$ cells $/ \mu \mathrm{L}, P=0.0233$; CD $41^{+} / \mathrm{CD} 42 \mathrm{a}^{+}$megakaryocytes/ $\mu \mathrm{L}$ [mean $\left.\pm \mathrm{SEM}\right]: 63 \pm 11$ cells $/ \mu \mathrm{L}$ vs. $25 \pm 4$ cells $/ \mu \mathrm{L}, P=0.0312$, respectively) (Online Supplementary Figure $S 3 A, B)$. Interestingly, the ratio of mature cells was similar (ratio of mature/total megakaryocytes, $[$ mean \pm SEM]: $0.91 \pm 0.03$ vs. $0.91 \pm 0.05 ; P=0.9540$ ) (Online Supplementary Figure S3C). Importantly, fewer platelets were released into the supernatant after megakaryocyte desialylation (platelets/ $\mu \mathrm{L}$ [mean \pm SEM]: $11 \pm 2$ cells $/ \mu \mathrm{L}$ vs. $4 \pm 1$ cells $/ \mu \mathrm{L} ; \mathrm{P}=0.0384$ ) (Online Supplementary Figure $S 3 D$ ). These results suggest that sialylation might be 
important for the differentiation of $\mathrm{CD}^{+} 4^{+}$cells into mature megakaryocytes.

\section{Discussion}

Thrombocytopenia in ITP patients is thought to be due to multiple alterations of the immune system leading to increased platelet destruction and impaired thrombopoiesis. ${ }^{2,25,30,31}$ In this report, we describe novel effector functions in which desialylating $\mathrm{AAb}$ from ITP patients interfere with platelet lifespan and function. First, we confirm that desialylation contributes to AAb-mediated destruction of human platelets and uncover a new mechanism, in which IgG anti-GPIIb/IIIa AAb induce crosslinking of Fcy RIIA leading to the release of sialidase from
A

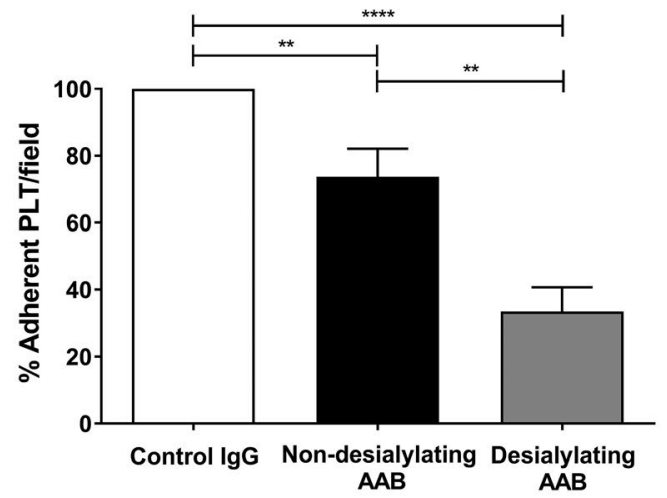

Fibrinogen

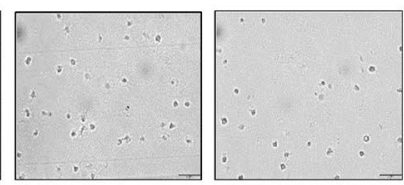

B

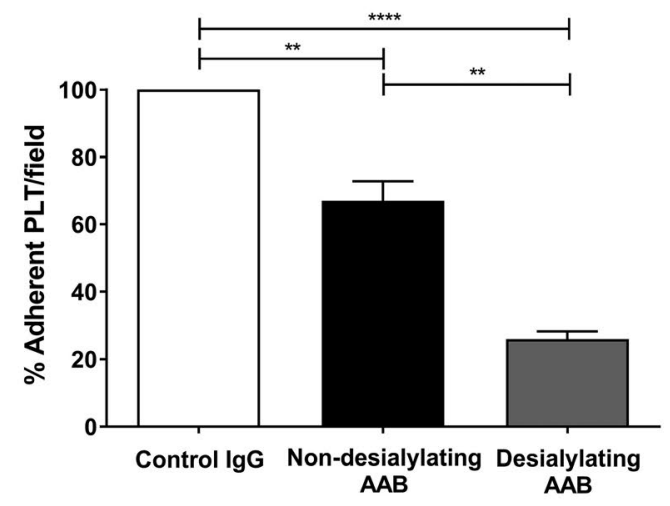

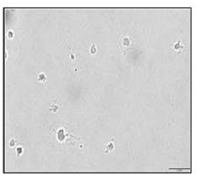

D
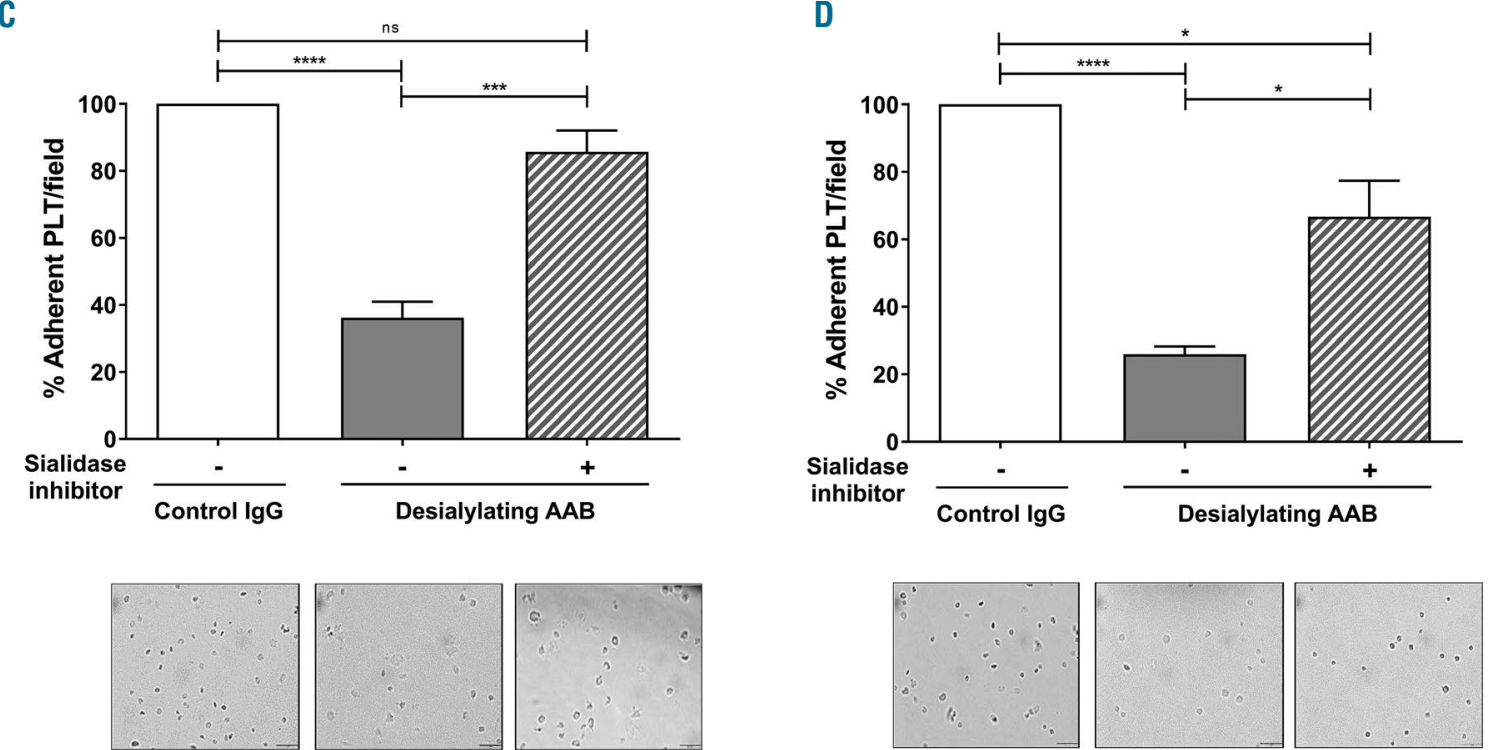

Figure 4. The impact of desialylating autoantibodies on platelet function. Washed platelets were preincubated with immunoglobulin $\mathrm{G}$ (IgG) fractions from healthy donors (white columns), patients with immune thrombocytopenia (ITP) with non-desialylating (black columns) and ITP patients with desialylating (gray columns) autoantibodies (AAB). Cells were then seeded on slides coated with fibrinogen (A) or von Willebrand factor (VWF, B). (C, D) To verify the role of desialylation in AABinduced impairment of platelet function, cells were preincubated with buffer (filled gray columns) or sialidase inhibitors (gray-white columns) prior to platelet adhesion to slides coated with fibrinogen (C) or VWF (D). Photographs of randomly chosen fields were captured to calculate the percentage of adherent cells compared to those in the control condition (considered as 100\%). The number of adherent platelets in the sample that was incubated with IgG from healthy donor in each individual experiment was considered $100 \%$. Data are shown as the mean of the percentage of adherent platelets \pm standard error of mean in the presence of AAB from three different ITP patients tested in three independent experiments with platelets from three different donors; ns, not significant, $* P<0.05, * * P<0.01, * * * P<0.001$ and $* * * * P<0.0001$. Images from representative fields of platelets adhered to fibrinogen or VWF, in the presence of control IgG (left panel) or ITP AAB (middle and right panels), respectively, are shown (scale bar: $10 \mu \mathrm{m})$. PLT: platelet. 
human platelets. Second, our data demonstrate that desialylation induced by AAb causes significant impairment of platelet function. This finding seems to have considerable relevance, since bleeding was observed more frequently in ITP patients with desialylating AAb in our clinical study. Most importantly, and to the best of our knowledge, we provide for the first time evidence that AAb-mediated desialylation inhibits megakaryocyte adhesion to bone marrow extracellular matrix proteins, leading to limited cell differentiation and reduced ability to form proplatelets.

The central role of AAb produced by B cells in ITP has been clearly established. ${ }^{15,31}$ In contrast, the exact mechanisms by which $\mathrm{AAb}$ interfere with platelet lifespan are still a matter of research. According to the current under- standing of ITP, AAb-mediated platelet destruction can be caused in one or more ways, such as phagocytosis, apoptosis, or complement activation. ${ }^{28,32,33}$ While these mechanisms are related to the Fc-mediated effector functions of the $\mathrm{AAb}$, recent studies proposed desialylation as an Fcindependent pathway of platelet elimination. ${ }^{9,34,35}$ Although AAb against GPIb/IX are generally considered to be the major mediators of platelet desialylation, ${ }^{36}$ systematic clinical studies to analyze the impact of glycoprotein specificity are rare. ${ }^{37,38}$ In contrast to previous studies, in our study desialylation was frequently observed in association with AAb against GPIIb/IIIa. Our data show that anti-GPIIb/IIIa-induced desialylation is mediated by Fcy RIIA crosslinking. $F\left(a b^{\prime}\right)$, fragments lacking the Fc-domain
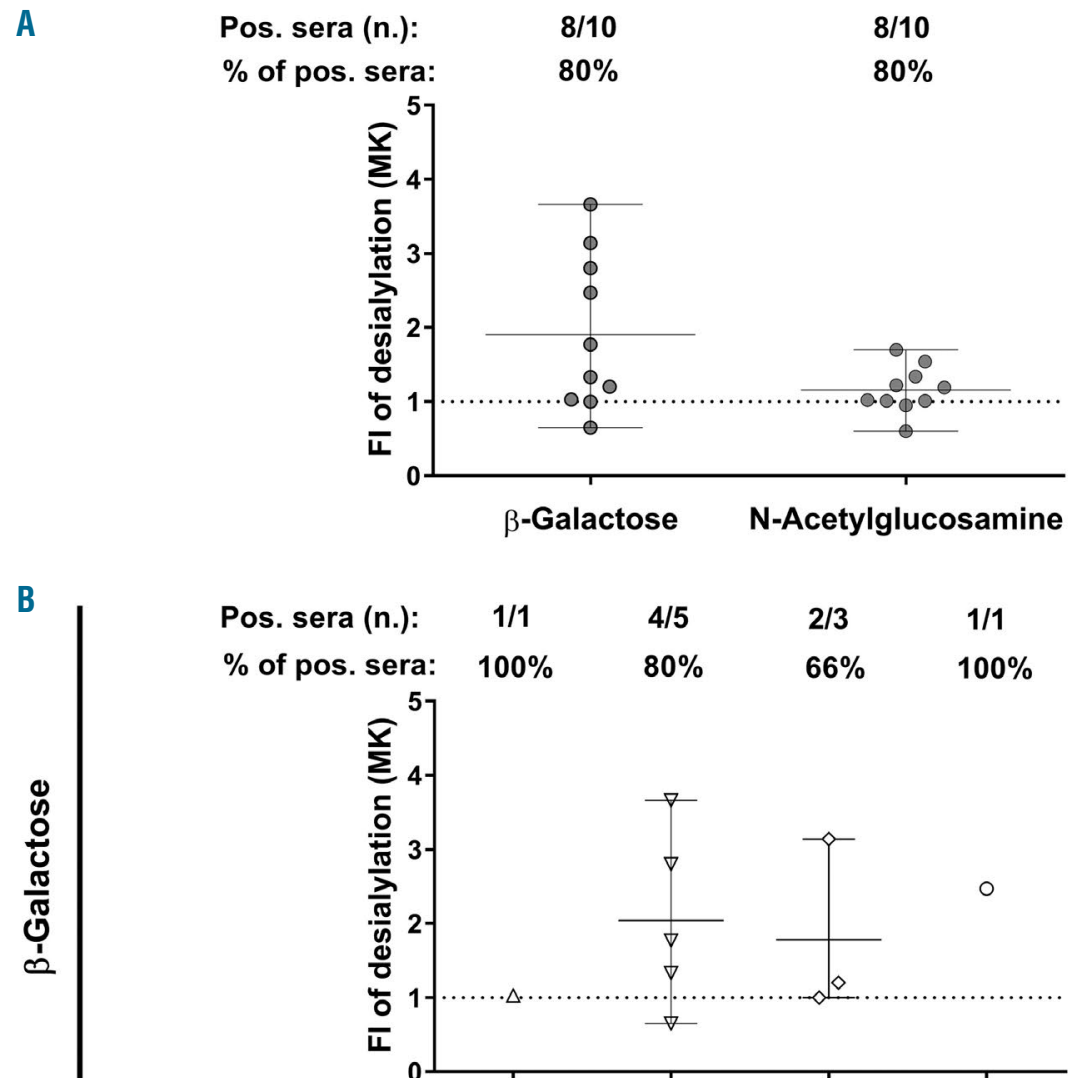

AAB-specificity: GP lb/lX GP Ilb/llla GP $\| b / l l l a$ No AAB $+\mid \mathrm{b} / \mathrm{IX}$

$\begin{array}{llclc}\text { Pos. sera (n.): } & 0 / 1 & 5 / 5 & 2 / 3 & 1 / 1 \\ \text { \% of pos. sera: } & 0 \% & 100 \% & 66 \% & 100 \%\end{array}$

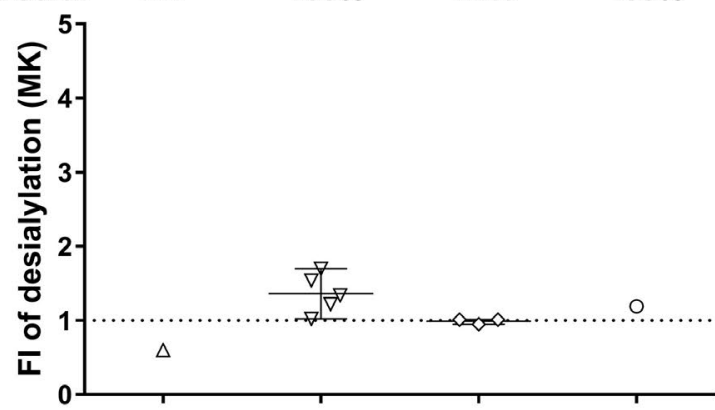

AAB-specificity: GP Ib/IX GP Ilb/llla GP Ilb/llla No AAB $+\mid \mathrm{lb} / \mathrm{IX}$

Figure 5. The ability of autoantibodies to desialylate CD34+-derived megakaryocytes. (A) CD34+-derived megakaryocytes were treated with immunoglobulin $\mathrm{G}$ (IgG) fractions from ten sera containing platelet-desialylating autoantibodies (AAB). Cleavage of sialic acid and exposure of $\beta$-galactose and $\mathrm{N}$-acetylglucosamine on megakaryocytes were measured by flow cytometer as fold increase compared to that produced with control IgG. $(B, C)$ The distribution of desialylating $A A B$ according to the glycoprotein specificity, as determined in a monoclonal antibody-specific immobilization of platelet antigens (MAIPA) assay. Each IgG sample was evaluated in three independent experiments using three different cell lines. The values presented are the mean and range. Fl: fold increase; GP: glycoprotein. 
showed no desialylation ability (data not shown). Of note, monoclonal antibodies against GPIIb/IIIa failed to cause desialylation of murine platelets. One possible explanation for this discrepancy is that murine platelets, in contrast to human platelets, do not express the Fcy RIIA on their surface. In order to investigate the biological relevance of human platelet desialylation, we first used the NSG animal model of passive immune thrombocytopenia. In this model, all tested desialylating AAb were efficient in removing human platelets from mouse circulation, regardless of their glycoprotein specificity. Importantly, when sialidase was inhibited, platelet survival was significantly enhanced. Considering that ITP AAb have been shown to be capable of inducing platelet apoptosis and a subgroup of them concomitantly desialylation, ${ }^{39-41}$ we decided to analyze the impact of desialylating $\mathrm{AAb}$ on platelet apoptosis. In our study, we observed that $40 \%$ of desialylating ITP AAb induced apoptosis signals in test platelets. However, our data showed no statistically significant correlation between the ability of desialylation and apoptosis, suggesting that ITP AAb-mediated desialylation may contribute to the impaired lifespan of platelets in an apoptosis-independent way.

In addition to accelerated platelet clearance, accumulat- ing evidence suggests that $\mathrm{AAb}$ are responsible for abnormal platelet production from megakaryocytes in ITP patients. ${ }^{30}$ Recent studies have shown that ITP patients have significantly fewer proplatelet-forming megakaryocytes compared to healthy controls, ${ }^{42}$ and that AAb have the ability to inhibit proplatelet formation by megakaryocytes. ${ }^{12}$ Moreover, animal studies indicate that antiplatelet $\mathrm{AAb}$ impair migration and adhesion of megakaryocytes without affecting these cells' viability and proliferation, ${ }^{43}$ or their number in the bone marrow. ${ }^{44}$ In our study, desialylating AAb from ITP patients were able to induce cleavage of sialic acid from the surface of megakaryocytes leading to a significant reduction in their ability to adhere to extracellular matrix proteins, fibrinogen and VWF, in a sialylation-dependent manner. Abnormal interactions between these glycoprotein receptors of the megakaryocytic lineage and their corresponding extracellular matrix ligands are thought to be responsible for impaired megakaryocyte development and reduced platelet production as, for example, shown in patients carrying functional mutations in GPIIb/IIIa ${ }^{45}$ and GPIb/IX. ${ }^{45,46}$ In addition, data from ex vivo models of thrombopoiesis demonstrated that the binding of fibrinogen and VWF to GPIIb/IIIa and GPIb/IX, respectively, enhances proplatelet production. ${ }^{29}$

A

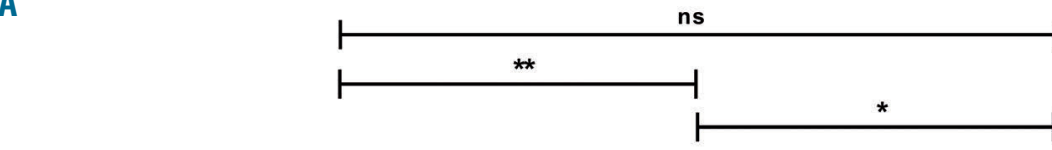

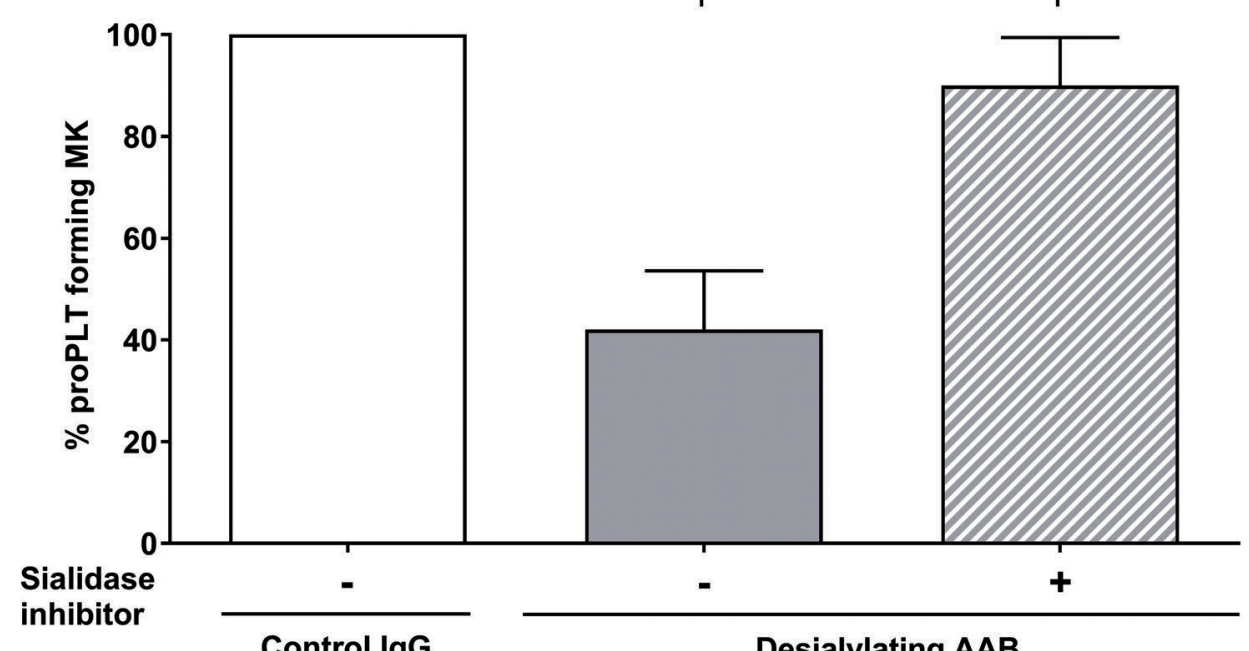

B
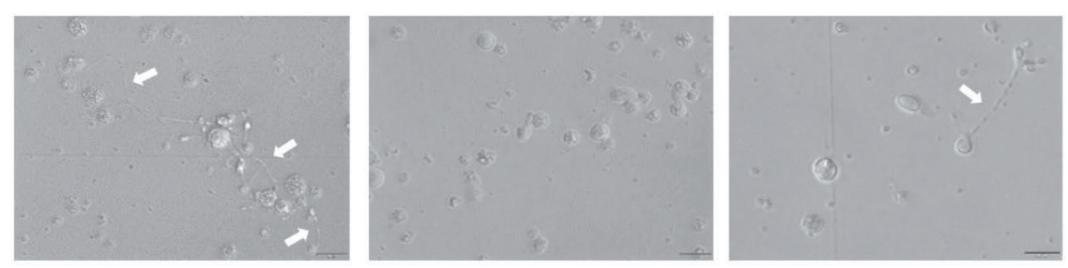

C
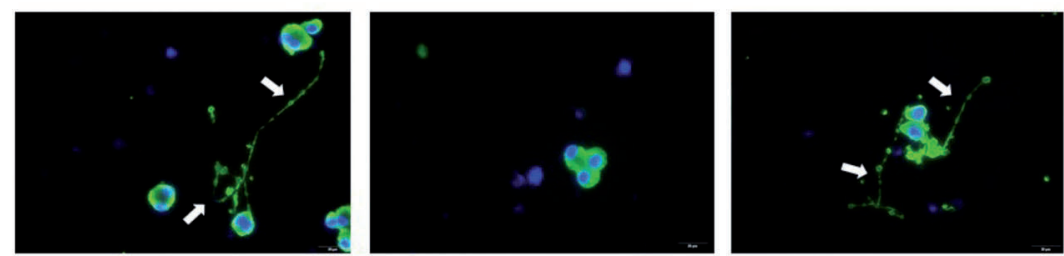

Figure 6. The impact of desialylating autoantibodies on proplatelet formation. (A) After 14 days of differentiation, megakaryocytes were cultured for 24 hours in the presence of immunoglobulin fractions from healthy donors (white column) or desialylating autoantibodies (AAB, gray column). To test the impact of desialylation on proplatelet formation, cells were treated with a sialidase inhibitor prior to their incubation with desialylating $\mathrm{AAB}$ (graywhite column). The percentage of proplatelet-forming megakaryocytes was determined as the number of megakaryocytes presenting at least one cytoplasmic projection with respect to the total number of megakaryocytes. Results were normalized to control immunoglobulin G (IgG) as $100 \%$. IgG samples from three different patients with immune thrombocytopenia were evaluated in three independent experiments using three different megakaryocyte cell lines and the mean values are shown. (B, C) Representative bright field (B) and immune fluorescence microscopic images $(C)$ of megakaryocyte-forming proplatelet extensions in the presence of IgG fractions from healthy donors (left panel), desialylating $A A B$ (middle) or in the presence of a sialidase inhibitor (right panel). Arrowheads indicate proplatelet extensions generated from megakaryocytes. Green staining, filamin; blue staining, nuclei. Scale bar: 20 um. The data shown are the mean \pm standard error of mean; ns, not significant, $* P<0.05$ and $* * P<0.01$. proPLT: proplatelet; $\mathrm{MK}$ : megakaryocyte. 
A recently published report described that $\mathrm{AAb}$ from ITP patients interfere with the interactions of megakaryocytes with different components of the bone marrow extracellular microenvironment, leading to reduced proplatelet formation. ${ }^{47}$ Our study confirms this observation and uncovers a new mechanism for the impaired thrombopoiesis in ITP. Desialylating AAb caused a significant reduction of proplatelets by inducing the cleavage of sialic acid from the megakaryocyte surface. We also found that sialylation is important for the differentiation of megakaryocytes. Along the same line, lower platelet counts were found in ITP patients with desialylating AAb, suggesting that not only may platelet elimination be increased in this group of patients, but thrombopoiesis might also be more severely affected. Of note, data from a recent clinical study showed that oseltamivir, a sialidase inhibitor, improved the response to thrombopoietin agonists in more than half of ITP patients with AAb against GPIb/IX. ${ }^{36}$ The concept of preventing desialylation in order to improve thrombopoiesis in ITP should be verified in the future.

Of note, reduced sialylation of serum IgG has been observed in autoimmune diseases such as rheumatoid arthritis and systemic lupus erythematosus. ${ }^{48}$ In our study, the exposure of $\beta$-galactose and $\mathrm{N}$-acetylglucosamine was reduced when platelets were preincubated with a sialidase inhibitor, indicating that the cleavage of sialic acid through endogenous sialidase is responsible for the increased lectin binding in our test system. This finding makes it very unlikely that our observation was modulated by the sialylation status of the AAb.

Although thrombocytopenia is considered to be a general feature in ITP, the clinical manifestations of the disease are very heterogeneous, with no biomarker available to predict the risk of bleeding. The presence of $\mathrm{AAb}$ might influence the clinical picture in ITP patients not only by causing a lower platelet count but also by reducing platelet responsiveness. In fact, functional platelet defects were observed in ITP patients with a bleeding tendency. ${ }^{49,50}$ In our study, desialylating $\mathrm{AAb}$ caused impairment of platelet adhesion to fibrinogen as well as VWF suggesting a possible impact on the hemostatic function of platelets. It is worth noting that a greater bleeding tendency was observed in ITP patients with desialylating AAb than in those with $A A b$ that failed to induce cleavage of sialic acid from the surface of platelets. Although prospective studies are still needed to verify a potential correlation, this finding may indicate a clinical relevance of our laboratory observations, suggesting that the treatment defined for each patient must take into consideration all of these aspects. Furthermore, our data as well as those from other recent studies ${ }^{36,41}$ suggest that a combination of therapies targeting the different pathological mechanisms, including platelet desialylation, by using, for example, a sialidase
A

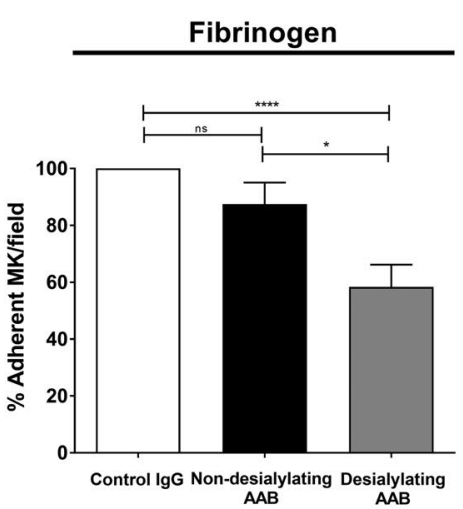

D

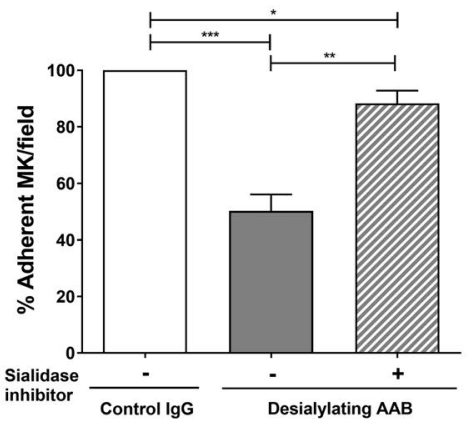

B

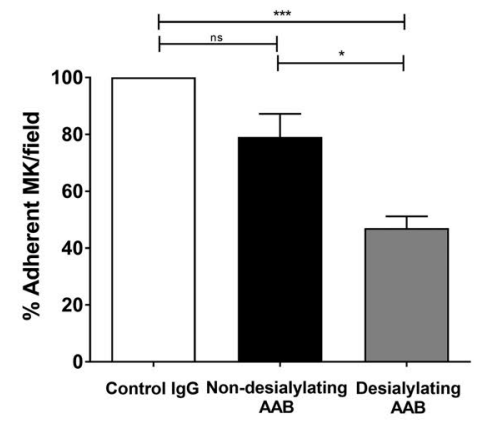

E

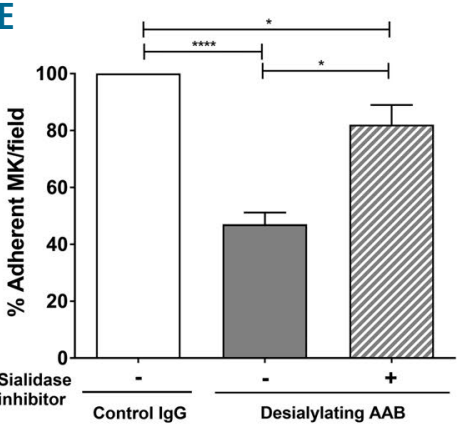

C

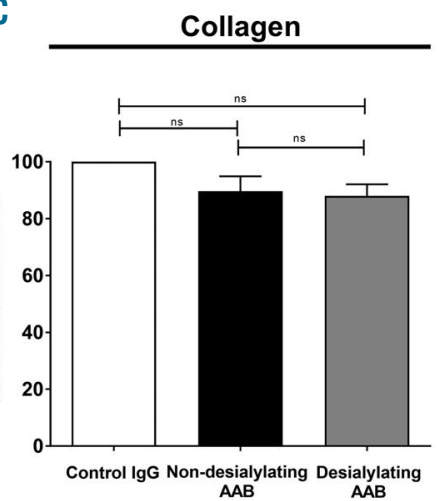

$\mathrm{F}$

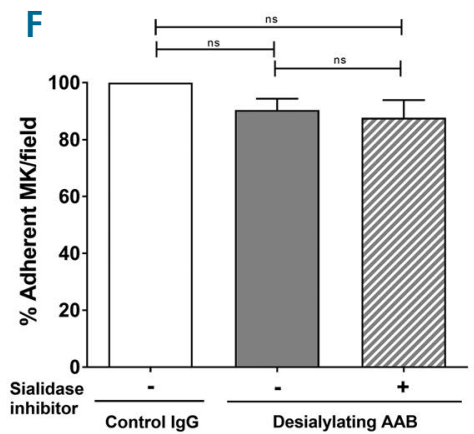

Figure 7. Interference of desialylating autoantibodies with the interaction between megakaryocytes and bone marrow extracellular matrix proteins. (A-C) Megakaryocytes were seeded on fibrinogen (A), von Willebrand factor (VWF, B) or collagen (C) -coated coverslips in the presence of immunoglobulin G fractions from healthy donors (white columns), non-desialylating (black columns) or desialylating (gray columns) autoantibodies (AAB). Photographs of randomly chosen fields were captured to calculate the percentage of adherent cells compared to that in the control condition (considered as 100\%). (D-F) To verify the role of desialylation in the impairment of megakaryocyte adhesion, a sialidase inhibitor (gray-white columns) was added to the cell culture prior to the incubation with AAB. Cells were then seeded on fibrinogen (D), VWF (E) or collagen (F)-coated coverslips. Data shown are the mean of the percentage adherent megakaryocytes \pm standard error of mean for AAB from three different patients with immune thrombocytopenia tested in three independent experiments with three different cell lines; ns, not significant, * $P<0.05$, $* * P<0.01, * * * P<0.001$ and $* * * * P<0.0001$. MK: megakaryocyte; IgG: immunoglobulin G. 
inhibitor, might be a more efficient clinical approach producing better, prolonged outcomes.

Since our study was retrospective and no follow-up data were available, we cannot draw definitive conclusions on the impact of desialylating $\mathrm{AAb}$ on response to therapy and disease progression or on the explicit effect of megakaryocyte desialylation on thrombopoiesis in ITP. The final proof of our concept regarding the contribution of AAb-mediated desialyation to the clinical picture in patients with ITP can only be provided by larger prospective clinical studies.

In conclusion, our results indicate that desialylation plays an important role in platelet destruction and impaired thrombopoiesis in ITP; and demonstrate that receptor sialyation status is of considerable functional relevance, since its changes not only affect platelet survival but also interfere with megakaryocyte differentiation and platelet generation. These novel findings highlight the multiple effects of AAb in ITP and add to the existing evidence that ITP is a group of disorders rather than one disease sharing common characteristics, namely loss of immune tolerance toward platelet and megakaryocyte antigens and impaired platelet lifespan, leading to thrombocytopenia with increased bleeding tendency.

\section{Disclosures}

No conflicts of interests to disclose.

\section{Contributions}

I.M. and TB designed the studv; IM, JZ, KA, LP, and TB performed the experiments; IM, JZ,SH, WB, CF UH KA and $T B$ collected and analyzed the data, interpreted the results and wrote the manuscript. All authors read and approved the manuscript.

\section{Acknowledgments}

The authors would like to thank Flavianna Rigoni for her excellent technical assistance and Stephen Bosher a native English speaker, for editing the manuscript. This work was supported by a research grant from the Medical Faculty of Tübingen, University Hospital of Tübingen, Germany, to IM and TB as well as one from Karina Althaus (TÜFF-Program 2563-0-0).

\section{References}

1. Provan D, Stasi R, Newland AC, et al. International consensus report on the investigation and management of primary immune thrombocytopenia. Blood. 2010;115(2):168-186.

2. Neunert C, Lim W, Crowther M, et al. The American Society of Hematology 2011 evidence-based practice guideline for immune thrombocytopenia. Blood. 2011;117(16): 4190-4207.

3. Moulis G, Palmaro A, Montastruc JL, Godeau B, Lapeyre-Mestre M, Sailler L. Epidemiology of incident immune thrombocytopenia: a nationwide population-based study in France. Blood. 2014;124(22):33083315 .

4. Bennett CM, Neunert C, Grace RF, et al. Predictors of remission in children with newly diagnosed immune thrombocytopenia: data from the Intercontinental Cooperative ITP Study Group Registry II participants. Pediatr Blood Cancer. 2018;65(1).

5. McKenzie CG, Guo L, Freedman J, Semple JW. Cellular immune dysfunction in immune thrombocytopenia (ITP). Br J Haematol. 2013;163(1):10-23

6. Shulman NR, Marder VJ, Weinrach RS. Similarities between known antiplatelet antibodies and the factor responsible for thrombocytopenia in idiopathic purpura. Physiologic, serologic and isotopic studies. Ann N Y Acad Sci. 1965;124(2):499-542.

7. Rank A, Weigert O, Ostermann H. Management of chronic immune thrombocytopenic purpura: targeting insufficient megakaryopoiesis as a novel therapeutic principle. Biologics. 2010;4:139-145.

8. Ku FC, Tsai CR, Der Wang J, Wang CH, Chang TK, Hwang WL. Stromal-derived factor-1 gene variations in pediatric patients with primary immune thrombocytopenia. Eur J Haematol. 2013;90(1):25-30.

9. Li J, van der Wal DE, Zhu G, et al. Desialylation is a mechanism of Fc-independent platelet clearance and a therapeutic target in immune thrombocytopenia. Nat Commun. 2015;6:7737.
10. Vianelli N, Galli M, de Vivo A, et al. Efficacy and safety of splenectomy in immune thrombocytopenic purpura: long-term results of 402 cases. Haematologica. 2005;90(1):72-77.

11. Lev PR, Grodzielski M, Goette NP, et al. Impaired proplatelet formation in immune thrombocytopenia: a novel mechanism contributing to decreased platelet count. $\mathrm{Br} \mathrm{J}$ Haematol. 2014;165(6):854-864.

12. Iraqi M, Perdomo J, Yan F, Choi PY, Chong $\mathrm{BH}$. Immune thrombocytopenia: antiplatelet autoantibodies inhibit proplatelet formation by megakaryocytes and impair platelet production in vitro. Haematologica. 2015;100(5):623-632.

13. Thon JN, Montalvo A, Patel-Hett S, et al. Cytoskeletal mechanics of proplatelet maturation and platelet release. J Cell Biol. 2010;191(4):861-874

14. McMillan R, Wang L, Tomer A, Nichol J, Pistillo J. Suppression of in vitro megakaryocyte production by antiplatelet autoantibodies from adult patients with chronic ITP. Blood. 2004;103(4):1364-1369.

15. Kiefel V, Freitag E, Kroll H, Santoso S, Mueller-Eckhardt C. Platelet autoantibodies (IgG, IgM, IgA) against glycoproteins IIb/IIIa and $\mathrm{Ib} / \mathrm{IX}$ in patients with thrombocytopenia. Ann Hematol. 1996;72(4):280-285.

16. Kiefel V, Scheld S, Freitag E, Kroll H, Mueller-Eckhardt C. [Two modifications of MAIPA assays for the demonstration of platelet antibodies]. Beitr Infusionsther Transfusionsmed. 1994;32:237-239.

17. Kiefel V. Transfusionsmedizin und Immunhämatologie. Springer. 2010:p624.

18. Chen M, Yan R, Zhou K, et al. Akt-mediated platelet apoptosis and its therapeutic implications in immune thrombocytopenia. Proc Natl Acad Sci U S A. 2018;115(45):E10682E10691.

19. Marini I, Aurich K, Jouni R, et al. Cold storage of platelets in additive solution: the impact of residual plasma in apheresis platelet concentrates. Haematologica. 2019;104(1):207-214.

20. Bakchoul T, Fuhrmann J, Chong BH, Bougie $\mathrm{D}$, Aster R, Subcommittee on Platelet Immunology. Recommendations for the use of the non-obese diabetic/severe combined immunodeficiency mouse model in autoimmune and drug-induced thrombocytopenia: communication from the SSC of the ISTH. Thromb Haemost. 2015;13(5):872-875.

21. Fuhrmann J, Jouni R, Alex J, et al. Assessment of human platelet survival in the NOD/SCID mouse model: technical considerations. Transfusion. 2016;56(6):1370-1376.

22. Mattia G, Vulcano F, Milazzo L, et al. Different ploidy levels of megakaryocytes generated from peripheral or cord blood CD34+ cells are correlated with different levels of platelet release. Blood. 2002;99(3):888-897.

23. Marini I, Rigoni F, Zlamal J, et al. Blood donor-derived buffy coat to produce platelets in vitro. Vox Sang. 2020;115(1):94-102

24. Liu Y, Wang Y, Gao Y, et al. Efficient generation of megakaryocytes from human induced pluripotent stem cells using food and drug administration-approved pharmacological reagents. Stem Cells Transl Med. 2015;4(4):309-319.

25. Di Buduo CA, Alberelli MA, Glembostky AC, et al. Abnormal proplatelet formation and emperipolesis in cultured human megakaryocytes from gray platelet syndrome patients. Sci Rep. 2016;6:23213.

26. Peterson JA, Kanack A, Nayak D, et al. Prevalence and clinical significance of lowavidity HPA-1a antibodies in women exposed to HPA-1a during pregnancy. Transfusion. 2013;53(6):1309-1318.

27. Bakchoul T, Kubiak S, Krautwurst A, et al. Low-avidity anti-HPA-1a alloantibodies are capable of antigen-positive platelet destruction in the NOD/SCID mouse model of alloimmune thrombocytopenia. Transfusion. 2011;51(11):2455-2461.

28. Najaoui A, Bakchoul T, Stoy J, et al. Autoantibody-mediated complement activation on platelets is a common finding in patients with immune thrombocytopenic purpura (ITP). Eur J Haematol. 2012;88 (2):167-174

29. Balduini A, Pallotta I, Malara A, et al. Adhesive receptors, extracellular proteins and myosin IIA orchestrate proplatelet formation by human megakaryocytes. J Thromb Haemost. 2008;6(11):1900-1907.

30. Marini I, Bakchoul T. Pathophysiology of 
autoimmune thrombocytopenia: current insight with a focus on thrombopoiesis. Hamostaseologie. 2019;39(3):227-237.

31. Cines DB, Liebman HA. The immune thrombocytopenia syndrome: a disorder of diverse pathogenesis and clinical presentation. Hematol Oncol Clin North Am. 2009;23(6):1155-1161.

32. Tsubakio T, Tani P, Curd JG, McMillan R. Complement activation in vitro by antiplatelet antibodies in chronic immune thrombocytopenic purpura. Br J Haematol. 1986;63(2):293-300.

33. Peerschke EI, Andemariam B, Yin W, Bussel JB. Complement activation on platelets correlates with a decrease in circulating immature platelets in patients with immune thrombocytopenic purpura. Br J Haematol. 2010;148(4):638-645.

34. Yan R, Chen M, Ma N, et al. Glycoprotein $\mathrm{Ib} \alpha$ clustering induces macrophage-mediated platelet clearance in the liver. Thromb Haemost. 2015;113(1):107-117.

35. Quach ME, Dragovich MA, Chen W, et al. Fc-independent immune thrombocytopenia via mechanomolecular signaling in platelets. Blood. 2018;131(7):787-796.

36. Revilla N, Corral J, Minano A, et al. Multirefractory primary immune thrombocytopenia; targeting the decreased sialic acid content. Platelets. 2019;30(6):743-751.

37. Tao L, Zeng Q, Li J, et al. Platelet desialylation correlates with efficacy of first-line therapies for immune thrombocytopenia. J Hematol Oncol. 2017;10(1):46.
38. Peng J, Ma SH, Liu J, et al. Association of autoantibody specificity and response to intravenous immunoglobulin $G$ therapy in immune thrombocytopenia: a multicenter cohort study. J Thromb Haemost. 2014;12(4):497-504.

39. Mason KD, Carpinelli MR, Fletcher JI, et al. Programmed anuclear cell death delimits platelet life span. Cell. 2007;128(6):11731186.

40. Deng G, Yu S, Li Q, et al. Investigation of platelet apoptosis in adult patients with chronic immune thrombocytopenia. Hematology. 2017;22(3):155-161.

41. Grodzielski M, Goette NP, Glembotsky AC, et al. Multiple concomitant mechanisms contribute to low platelet count in patients with immune thrombocytopenia. Sci Rep 2019;9(1):2208.

42. Riviere E, Viallard JF, Guy A, et al. Intrinsically impaired platelet production in some patients with persistent or chronic immune thrombocytopenia. Br J Haematol. 2015;170(3):408-415.

43. Zeng DF, Chen F, Wang $S$, et al Autoantibody against integrin $\alpha v \beta 3$ contributes to thrombocytopenia by blocking the migration and adhesion of megakaryocytes. J Thromb Haemost. 2018;16(9): 1843-1856.

44. Guo L, Kapur R, Aslam R, et al. Antiplatelet antibody-induced thrombocytopenia does not correlate with megakaryocyte abnormalities in murine immune thrombocytopenia. Scand J Immunol. 2018;88(1):e12678.
45. Kunishima S, Kashiwagi H, Otsu M, et al. Heterozygous ITGA2B R995W mutation inducing constitutive activation of the $\alpha I$ Ib $\beta 3$ receptor affects proplatelet formation and causes congenital macrothrombocytopenia. Blood. 2011;117 (20):5479-5484.

46. Strassel C, Eckly A, Leon C, et al. Intrinsic impaired proplatelet formation and microtubule coil assembly of megakaryocytes in a mouse model of Bernard-Soulier syndrome. Haematologica. 2009;94(6):800-810.

47. Grodzielski M, Di Buduo CA, Goette NP, et al. Autoantibodies in immune thrombocytopenia affect the physiological interaction between megakaryocytes and bone marrow extracellular matrix proteins. Br J Haematol. 2018;183(2):319-323.

48. Seeling M, Bruckner C, Nimmerjahn F. Differential antibody glycosylation in autoimmunity: sweet biomarker or modulator of disease activity? Nat Rev Rheumatol. 2017;13(10):621-630.

49. van Bladel ER, Laarhoven AG, van der Heijden LB, et al. Functional platelet defects in children with severe chronic ITP as tested with 2 novel assays applicable for low platelet counts. Blood. 2014;123(10):15561563.

50. Frelinger AL 3rd, Grace RF, Gerrits AJ, Carmichael SL, Forde EE, Michelson AD Platelet function in ITP, independent of platelet count, is consistent over time and is associated with both current and subsequent bleeding severity. Thromb Haemost. 2018;118(1):143-151. 